\title{
Avaliação do crescimento e desenvolvimento de crianças institucionalizadas
}

\author{
Evaluation of growth and development of institutionalized children \\ Evaluación del crecimiento y desarrollo de niños institucionalizados
}

\section{Caroline Magna Pessoa Chaves', Francisca Elisângela Teixeira Lima", Larissa Bento de Araújo Mendonça", Ires Lopes Custódio"', Érica Oliveira Matias"}

\author{
' Secretaria de Saúde do Estado do Ceará, Hospital Geral Dr. Waldemar Alcântara. Fortaleza-CE, Brasil. \\ "Universidade Federal do Ceará, Faculdade de Farmácia, Odontologia e Enfermagem, Departamento de Enfermagem. \\ Fortaleza-CE, Brasil. \\ II' Secretaria de Saúde do Estado do Ceará, Hospital Dr. Carlos Alberto Studart Gomes. Fortaleza-CE, Brasil.
}

\author{
Submissão: 29-08-2012 Aprovação: 08-08-2013
}

\begin{abstract}
RESUMO
O estudo objetivou avaliar o crescimento e desenvolvimento de crianças de 0 a 6 anos institucionalizadas. Abordagem descritiva, transversal e de natureza quantitativa. Foi realizado em um abrigo da Secretaria do Trabalho e Desenvolvimento Social do Governo do Estado do Ceará, nos meses de março e abril de 2011. A amostra foi composta por 44 crianças. Prevaleceram crianças de sexo masculino (59,1\%); faixa etária entre 24 a 72 meses (56,8\%), e tempo de abrigamento superior a um ano $(72,7 \%)$. Verificou-se que as crianças estavam na faixa de normalidade nutricional. Porém, 65,9\% das crianças não alcançaram pelo menos um dos marcos do desenvolvimento propostos pelo Ministério da Saúde. O estudo possibilitou uma reflexão sobre como as crianças residentes em abrigos estão sendo assistidas pela equipe de saúde, tornando-se necessário realizar o acompanhamento de seu crescimento e desenvolvimento, a fim de intervir sobre as alterações encontradas.

Descritores: Crescimento e Desenvolvimento; Institucionalização; Saúde da Criança.
\end{abstract}

ABSTRACT
The study aimed to evaluate the growth and development of institutionalized children from 0-6 years old. It had a descriptive, transversal and quantitative approach, and was held in a shelter from the Bureau of Labor and Social Development of the Government of the State of Ceará, in the months of March and April 2011. The sample comprised 44 children. It the sample prevailed male children (59.1\%), aged 24 to 72 months $(56.8 \%)$ and with time of sheltering more than one year $(72.7 \%)$. It was found that the children were in the normal range of nutrition; however, $65.9 \%$ of children did not achieve at least one of the development milestones proposed by the Ministry of Health. The study suggests a reflection on how children living in shelters are being assisted by the health team, making it necessary follow up their growth and development, in order to intervene on the alterations.

Key words: Growth and Development; Institutionalization; Child Health.

\section{RESUMEN}

El objetivo del estudio fue evaluar el crecimiento y desarrollo de niños de 0-6 años institucionalizados. Tuve un enfoque descriptivo, transversal y cuantitativo, y se llevó a cabo en un abrigo de la Secretaria de Trabajo y Desarrollo Social del Gobierno del Estado de Ceará, en los meses de marzo y abril de 2011. La muestra fue de 44 niños. Prevaleció en la muestra niños varones $(59,1 \%)$, con edades comprendidas entre 24 y 72 meses $(56,8 \%)$, y con un tiempo de albergue superior a un año $(72,7 \%)$. Se observó que los niños estaban en el estado de nutrición normal. Sin embargo, el 65,9\% de los niños no logró, al menos, uno de los hitos del desarrollo propuestos por el Ministerio de Salud. El estudio sugiere una reflexión sobre cómo los niños que viven en los refugios están siendo asistidos por el equipo de salud, por lo que es necesario el seguimiento de su crecimiento y desarrollo, a fin de intervenir en las alteraciones.

Palabras clave: Crecimiento y Desarrollo; Institucionalización; Salud del Niño.

\section{AUTOR CORRESPONDENTE Érica Oliveira Matias E-mail: erica_enfermagem@yahoo.com.br}




\section{INTRODUÇÃO}

O crescimento e desenvolvimento da criança é o principal indicador de suas condições de saúde. Assim, o Ministério da Saúde prioriza seu acompanhamento desde o nascimento até os dez anos de idade na atenção básica, por meio da consulta de puericultura, buscando detectar precocemente alterações no crescimento e desenvolvimento da criança para evitar complicações ${ }^{(1)}$.

O crescimento, de um modo geral, é considerado como aumento do tamanho corporal, que cessa com o término do aumento em altura. Assim, para avaliar o crescimento infantil é preciso realizar a mensuração e o acompanhamento das medidas antropométricas da criança, tais como: peso, estatura, perímetro cefálico e torácico, relacionando-os entre $\mathrm{si}^{(1)}$.

Em relação ao desenvolvimento, pode ser entendido como mudança e expansão graduais; progresso dos estágios mais simples aos mais avançados de complexidade; surgimento e expansão das capacidades do indivíduo por meio do crescimento, da maturidade e do aprendizado ${ }^{(2)}$.

Diante da interferência do ambiente de vivência da criança no crescimento e desenvolvimento infantil, a avaliação das crianças institucionalizadas deve ser sistemática e periódica. Tal fato destaca-se no Brasil, por haver um grande número de crianças abandonadas pelos pais, vivendo institucionalizadas em abrigos.

Uma pesquisa realizada pelo Instituto de Pesquisa Econômica Aplicada que seguiu as orientações do Comitê de Reordenamento da Rede Nacional de Abrigos para Infância e Adolescência buscou conhecer a situação de abrigos beneficiados com recursos do governo federal. Foram estudados cerca de 590 abrigos na rede nacional, e a maioria absoluta das crianças abrigadas tem família $(86,7 \%)$, cujo motivo mais citado da institucionalização foi a pobreza $(24,2 \%)$, tendo se destacado também outros motivos: abandono $(18,9 \%)$, violência doméstica $(11,7 \%)$, dependência química dos pais e responsáveis incluindo o alcoolismo $(11,4 \%)$, moradores de rua $(7 \%)$ e orfandade $(5 \%)^{(3)}$.

$\mathrm{O}$ ambiente institucional geralmente não oferece condições propícias para o pleno desenvolvimento de crianças, por uma série de razões, dentre as quais se podem citar a privação de contato íntimo com a família, elevado número de crianças por cuidadora, dificultando a estimulação do seu desenvolvimento e um elevado número de crianças por abrigo, acarretando em menor qualidade no cuidado e no estabelecimento de laços afetivos ${ }^{(4-5)}$.

Diante dessas considerações, busca-se responder aos seguintes questionamentos: o crescimento das crianças institucionalizadas está de acordo com os parâmetros do Ministério da Saúde? O desenvolvimento das crianças institucionalizadas condiz com os marcos de desenvolvimento infantil propostos pelo Ministério da Saúde?

Portanto, objetivou-se com este estudo avaliar o crescimento e desenvolvimento de crianças institucionalizadas, de 0 a 6 anos, comparando com os parâmetros propostos pelo Ministério da Saúde.

\section{METODOLOGIA}

Trata-se de um estudo descritivo, transversal e de natureza quantitativa, realizado em um abrigo da Secretaria do TrabaIho e Desenvolvimento Social do Governo do Estado do Ceará. A população foi constituída por 81 crianças, que residiam em tal instituição durante o período da pesquisa. A amostra foi composta por todas as crianças que atenderam aos seguintes critérios de inclusão: estar na faixa etária de 0 a 6 anos e não ser portadora de alterações físicas, tais como: distúrbios neurológicos, mentais, visuais, endócrinos ou auditivos, dentre outros que interfiram no crescimento e desenvolvimento infantil. Atenderam a esses critérios 44 crianças.

Dentre as crianças que não atenderam os critérios de inclusão, vinte apresentaram distúrbios neurológicos, seis eram portadores de malformações congênitas, uma apresentava neoplasia cerebral, oito ultrapassaram a faixa etária estabelecida e duas não possuíam data de nascimento conhecida.

A coleta de dados foi realizada nos meses de março e abril de 2011 por meio do preenchimento de um instrumento semiestruturado elaborado para consulta aos prontuários e preenchido pelos pesquisadores; e por meio da realização do exame físico na criança. Cada criança foi avaliada no consultório médico da própria instituição, quanto ao crescimento e desenvolvimento infantil (peso, comprimento, perímetro cefálico, perímetro torácico, marcos do desenvolvimento infantil), além dos reflexos primitivos em crianças menores de um ano. Os resultados foram analisados de forma descritiva e apresentados por meio de tabelas, fundamentados na literatura pertinente.

O estudo respeitou os princípios da resolução nº 196/1996, da Comissão Nacional de Saúde ${ }^{(6)}$. Para tanto, foi solicitado autorização da Diretora da instituição para o desenvolvimento do estudo, a qual assinou o Termo de Fiel Depositário e o Termo de Consentimento Livre e Esclarecido, tendo em vista que a mesma é responsável por todas as crianças do abrigo. Após, o projeto de pesquisa foi encaminhado ao Comitê de Ética em Pesquisa da Universidade Federal do Ceará, que recebeu parecer favorável para seu desenvolvimento, conforme Protocolo no $283 / 10$.

\section{RESULTADOS}

Foram analisadas ao todo 44 crianças abrigadas, na faixa etária de 0 a 6 anos, que não eram portadoras de alterações físicas, pois as mesmas poderiam interferir diretamente em seu crescimento e desenvolvimento.

Conforme mostra a tabela 1 , predominaram as seguintes características na amostra: sexo masculino (59,1\%); faixa etária entre 24 a 72 meses (56,8\%); e tempo de abrigamento superior a um ano $(72,7 \%)$.

As causas mais frequentes foram dependência química dos genitores (36,36\%) e o abandono (31,81\%). Esses dados comprovam que essas crianças são oriundas de uma camada frágil da população e que vivenciaram situações de risco para o seu crescimento e desenvolvimento físico, mental e social. Acredita-se que essas crianças viviam em ambientes inapropriados, marcados pela violência e negligência de seus cuidados. 
Tabela 1 - Caracterização das crianças ao quanto ao sexo, idade e tempo de abrigamento. Fortaleza-CE, 2011.

\begin{tabular}{|c|c|c|c|}
\hline Características das crianças & $n=44$ & $\%$ & Média/Desvio padrão \\
\hline \multicolumn{4}{|l|}{ Sexo } \\
\hline Feminino & 18 & 40,9 & \\
\hline Masculino & 26 & 59,1 & \\
\hline \multicolumn{4}{|l|}{ Idade } \\
\hline$<12$ meses & 9 & 20,5 & \\
\hline 12 a 24 meses & 10 & 22,7 & 31 meses/19 meses \\
\hline$>24$ meses a 72 meses & 25 & 56,8 & \\
\hline \multicolumn{4}{|l|}{ Tempo de abrigamento } \\
\hline$<6$ meses & 5 & 11,4 & \\
\hline 6 meses a 1 ano & 7 & 15,9 & 20 meses/ 14 meses \\
\hline 1 a 3 anos & 27 & 61,3 & \\
\hline$>3$ anos & 5 & 11,4 & \\
\hline
\end{tabular}

Tabela 2 - Distribuição das crianças quanto à causa do abrigamento. Fortaleza-CE, 2011.

\begin{tabular}{lcc}
\hline Motivo do abrigamento & $\mathbf{N}=\mathbf{4 4}$ & \% \\
Dependência química dos pais & 16 & 36,4 \\
Abandono & 14 & 31,8 \\
Moradores de rua & 6 & 13,6 \\
Genitores com transtorno Mental & 5 & 11,4 \\
Pobreza & 2 & 4,5 \\
Violência doméstica & 1 & 2,3 \\
\hline
\end{tabular}

\section{Avaliação do crescimento das crianças}

Abordando a relação peso/idade, percebeu-se que a maioria $(61,4 \%)$ estava na faixa de normalidade nutricional, seguida pelas crianças que estavam com peso baixo $(20,4 \%)$. Apenas $4,5 \%$ das crianças foram classificadas como de peso muito baixo e $2,3 \%$ foram incluídas na faixa de sobrepeso.

Tabela 3 - Caracterização das crianças quanto ao percentil do peso/idade. Fortaleza-CE, 2011.

\begin{tabular}{ccc}
\hline Relação Peso e Idade & $\mathbf{n = 4 4}$ & $\mathbf{\%}$ \\
$<$ Percentil 0,1 & 2 & 4,5 \\
$0,1<$ Percentil < & 9 & 20,4 \\
$3<$ Percentil < 10 & 5 & 11,4 \\
$3<$ Percentil < 97 & 27 & 61,4 \\
$>$ Percentil 97 & 1 & 2,3 \\
\hline
\end{tabular}

As crianças que se encontraram no percentil de normalidade nutricional também apresentaram normalidade estatural. Diante disso, percebe-se que o ambiente institucional oferece condições propícias para o crescimento das crianças, o que pode ser evidenciado pelo fato de haver o oferecimento de dieta balanceada e completa em nutrientes nesse local.
Tabela 4 - Caracterização das crianças quanto ao percentil estatura/idade e percentil peso/estatura. Fortaleza-CE, 2011.

\begin{tabular}{lcc}
\hline Estatura/Idade & $\mathbf{n = 4 4}$ & $\mathbf{\%}$ \\
$<$ percentil 3 & 12 & 27,3 \\
$3<$ percentil < 97 & 32 & 72,7 \\
Peso/Estatura & & \\
$<$ Percentil 3 & 2 & 4,6 \\
$3<$ Percentil < 97 & 37 & 84 \\
$\geq$ Percentil 97 & 5 & 11,4 \\
\hline
\end{tabular}

De acordo com a tabela 4 , pode-se visualizar que $72,7 \%$ das crianças apresentaram altura dentro dos limites esperados. Entretanto, foi evidenciada estatura baixa para a idade em $27,3 \%$ das crianças, a qual prevaleceu na fase pré-escolar.

Com relação ao percentil peso/estatura, constatou-se que grande parte das crianças encontrou-se na faixa de normalidade, totalizando $84 \%$. Contudo, $11,4 \%$ das crianças encontraram-se na faixa de sobrepeso e 4,6\% na faixa de desnutrição aguda.

Observou-se que das 37 crianças que se encontrara na faixa de normalidade no percentil peso/estatura, 11 apresentaram também déficit estatural, o que permite afirmar que pode estar havendo uma adequação do peso dessas crianças para uma estatura que não é normal. É preciso que haja uma assistência adequada a essas crianças, buscando corrigir os motivos que não estão sendo favoráveis ao seu crescimento linear.

\section{Avaliação do desenvolvimento das crianças}

Dentro dos aspectos avaliados do desenvolvimento infantil, têm-se os marcos de desenvolvimentos como aspectos a serem avaliados, os quais segundo o Ministério da Saúde, para cada intervalo de idade estabelecido, são atribuídos marcos, cada um abordando fatores maturativos, psicomotores, sociais e psíquicos ${ }^{(1)}$, conforme descritos a seguir: 
- Crianças de 0 a 1 mês: Na instituição não havia nenhuma criança nessa faixa etária, assim os reflexos presentes nesse período não foram avaliados.

- Crianças de 1 a 2 meses: Havia duas crianças na faixa etária de 1 a 2 meses, as quais foram avaliadas quanto aos seguintes marcos:

$\checkmark$ Fixa e acompanha objetos em seu campo visual e colocada de bruços, levanta a cabeça momentaneamente: as duas crianças responderam positivamente a esses marcos, observando atentamente os objetos coloridos, conseguiram levantar a cabeça por instantes quando posicionadas de bruços.

$\checkmark$ Arrulha e sorri espontaneamente: as duas crianças não arrulhavam espontaneamente e nem com estímulos, porém responderam ao sorriso, olhando atentamente para a examinadora.

$\checkmark$ Começa a diferenciar dia e noite: somente uma das crianças demonstrou permanecer mais acordada e disposta durante o dia, a outra criança permaneceu sonolenta logo após a avaliação.

- Crianças de 3 a 4 meses: Apenas uma criança avaliada apresentava a faixa etária de 3 a 4 meses, sendo avaliada quanto aos seguintes marcos:

$\checkmark$ Postura: passa da posição lateral para a linha média: a criança apresentou esse marco, trocava de posição várias vezes, foi ativa nos movimentos.

$\checkmark$ Colocada de bruços, levanta e sustenta a cabeça apoiando-se no antebraço: a criança demonstrou essa habilidade.

$\checkmark$ Emite sons, balbucia: não houve emissão de sons ou balbucios, apenas sorriso e olhar atento para o observador.

$\checkmark$ Conta com a ajuda de outra pessoa, mas não fica passiva: esse marco não foi encontrado, visto que o lactente permaneceu passivo durante toda a avaliação.

- Crianças de 5 a 6 meses: Também somente uma criança avaliada apresentava a faixa etária de 5 a 6 meses, a qual respondeu positivamente a todos os marcos de sua faixa etária, como estão citados a seguir:

$\checkmark$ Rola da posição supina para prona: o lactente movimentou-se ativamente tanto no berço quanto no chão.

$\checkmark$ Levantada pelos braços, ajuda com o corpo: a criança não se manteve passiva quando levantada.

$\checkmark$ Vira a cabeça na direção de uma voz ou objeto sonoro: mostrou-se atenta à fala do examinador, e também aos barulhos provocados com brinquedos.

$\checkmark$ Reconhece quando se dirigem a ela: ao chamá-la olhava logo em seguida, apresentando sorrisos e expressões faciais de satisfação.

- Crianças de 7 a 9 meses: Na faixa etária de 7 a 9 meses, duas crianças encontravam-se nessas idades, as quais foram avaliadas quanto aos seguintes marcos:

$\checkmark$ Senta-se sem apoio: as duas crianças já sentavam sem apoio e conseguiam arrastar-se. Uma delas tinha 8 meses de idade e se colocava de pé com apoio. $\checkmark$ Segura e transfere objetos de uma mão para a outra: elas demonstraram curiosidade com os objetos ao redor, esforçando-se para pegá-los, transferindo-os de uma mão para a outra.

$\checkmark$ Responde diferentemente a pessoas familiares ou a estranhos: nenhuma das duas respondeu diferentemente a estranhos. Ao chegar uma pessoa desconhecida para elas, logo respondiam com sorrisos.

$\checkmark$ Imita pequenos gestos ou brincadeiras: apenas uma criança imitava gestos e brincadeiras, a outra apenas olhava e sorria, mas não conseguia reproduzi-los.

- Crianças de 10 a 12 meses: Havia três crianças na faixa etária de 10 a 12 meses, as quais foram avaliadas quanto aos seguintes marcos:

$\checkmark$ Arrasta-se ou engatinha: as três crianças arrastavam-se, engatinhavam e se colocavam de pé quando apoiadas sobre móveis ou pessoas.

$\checkmark$ Pega objetos usando o polegar e o indicador: também quanto a esse marco todas responderam positivamente.

$\checkmark$ Emprega pelo menos uma palavra com sentido: nenhuma criança pronunciava qualquer palavra com sentido, apenas diziam sílabas, apontavam para o que queriam e balbuciavam.

$\checkmark$ Faz gestos com a mão e a cabeça (tchau, não, bate palmas, etc): todas as três crianças gesticulavam quando estimuladas.

- Crianças de 13 a 18 meses: Havia quatro crianças na faixa etária de 13 a 18 meses, as quais foram avaliadas quanto aos seguintes marcos:

$\checkmark$ Anda sozinha, raramente cai: apenas uma criança não andava.

$\checkmark$ Tira sozinha qualquer peça do vestuário: apenas uma criança demonstrou essa habilidade.

$\checkmark$ Combina pelo menos duas ou três palavras: somente uma criança pronunciava cerca de duas palavras, as outras não falavam qualquer palavra.

$\checkmark$ Distancia-se da mãe sem perdê-la de vista: nesse caso foram consideradas como mãe as cuidadoras. Todas as crianças distanciavam-se das cuidadoras, mas sempre olhando em busca delas.

- Crianças de 19 meses a 2 anos: Havia cinco crianças na faixa etária de 19 meses a 2 anos, as quais foram avaliadas quanto os seguintes marcos:

$\checkmark$ Leva os alimentos à boca, com sua própria mão: foi percebido que todas elas demonstraram essa habilidade.

$\checkmark$ Corre e/ou sobe degraus baixos: todas as crianças movimentaram-se ativamente, correndo, pulando, subindo degraus.

$\checkmark$ Aceita a companhia de outras crianças, mas brinca isoladamente: observou-se que todas as crianças brincavam umas com as outras, e em instantes brincaram sozinhas também.

$\checkmark$ Diz seu próprio nome e nomeia objetos como sendo seu: nenhuma criança dizia seu próprio nome ou nomeava objetos como sendo seu. Foi visto que elas pronunciavam palavras como: tia, neném, tchau. 
- Crianças de 2 a 3 anos: Havia oito crianças na faixa etária de 2 a 3 anos de idade, as quais foram avaliadas quanto os seguintes marcos:

$\checkmark$ Veste-se com auxílio: somente três crianças apresentaram esse marco.

$\checkmark$ Fica sobre um pé, momentaneamente: todas as crianças apresentaram essa habilidade.

$\checkmark$ Usa frases: apenas uma criança usava frases, as outras pronunciavam palavras isoladas.

$\checkmark$ Começa o controle esfincteriano: nenhuma das crianças possuía o controle dos esfíncteres, todas elas permaneciam durante todo o dia de fraldas de algodão, sendo percebida a prática de realizarem as eliminações vesicais e intestinais na própria fralda.

- Crianças de 3 a 4 anos: Havia sete crianças na faixa etária de 3 a 4 anos de idade, as quais foram avaliadas quanto os seguintes marcos:

$\checkmark$ Reconhece mais de duas cores: somente uma criança estava com esse desenvolvimento adequado, pois conseguia distinguir mais de duas cores. As outras cinco crianças ainda não haviam apresentado esse marco.

$\checkmark$ Pula sobre um pé só e brinca com outras crianças: todas as crianças apresentaram esse marco.

$\checkmark$ Imita pessoas da vida cotidiana (pai, mãe, médico): somente três crianças demonstraram a prática de imitar os cuidadores, colegas, professores da escola ou funcionários do abrigo.

- Crianças de 4 a 6 anos: A faixa etária de 4 a 6 anos foi a de maior predomínio nas crianças participantes do estudo, sendo representada por 11 crianças, as quais foram avaliadas quanto aos seguintes marcos:

$\checkmark$ Veste-se sozinha: quatro crianças não demonstraram essa habilidade.

$\checkmark$ Pula alternadamente com um e outro pé e alterna momentos cooperativos com agressivos: foram observados esses comportamentos em todas as crianças.

$\checkmark$ Capaz de expressar preferências e ideias próprias: apenas uma criança demonstrou dificuldade em expressar suas preferências, ela não demonstrou interesse em falar de suas idéias.

\section{DISCUSSÃO}

Como se observa em pesquisa mais completa e recente sobre o assunto, realizada pelo Instituto de Pesquisa Econômica Aplicada $^{(3)}$, que analisou 626 instituições de abrigo em todas as regiões brasileiras, a maioria das crianças abrigadas são do sexo masculino (58,5\%), encontram-se após a primeira infância $(61,3 \%)$ e a parcela mais significativa está nos abrigos há um período entre dois e cinco anos.

De acordo com o estudo realizado no abrigo em Fortaleza, e a pesquisa desenvolvida pelo IPEA ${ }^{(3)}$, pode-se afirmar que os dados são correspondentes, nos principais pontos analisados: sexo, idade e tempo de institucionalização. Isso indica que o perfil das crianças abrigadas na instituição em estudo assemelhou-se com o das diferentes regiões brasileiras.
Mediante esses dados pode-se constatar também uma permanência inadequada dessas crianças nos abrigos, contrariando as normas do Estatuto da Criança e do Adolescente ${ }^{(7)}$, o qual afirma ser o abrigo uma medida provisória e excepcional, utilizável como forma de transição para a colocação em família substituta, não implicando privação de liberdade.

Uma pesquisa desenvolvida com 127 crianças comprovou que o abrigamento deveria ser temporário, ocorrendo até que melhores condições fossem oferecidas às crianças, no leito da família ou nos casos de adoção, já que são fatores de risco para o seu pleno desenvolvimento psíquico, interferindo assim no seu crescimento ${ }^{(8)}$.

Em estudo bibliográfico com enfoque nos fatores que interferem no crescimento de crianças nos primeiros anos, evidenciou-se a importância dos fatores extrínsecos como variáveis biológicas, fatores socioeconômicos, culturais, educacionais, entre outros, exerce grande influência para a ocorrência de déficits nutricionais e estaturais ${ }^{(9)}$.

Resultados semelhantes foram encontrados em estudo que realizou uma avaliação antropométrica transversal e descritiva em quatro creches de Minas Gerais com crianças de baixo nível socioeconômico, entre 16 e 82 meses de idade, no qual se observou que a maioria das crianças apresentou bom estado nutricional. Ou seja, eram eutróficas e possuíam peso e altura adequados para idade ${ }^{(10)}$.

Porém, destaca-se que houve divergência entre as classificações das crianças avaliadas no presente estudo quanto ao peso para idade, embora elas habitem em uma mesma instituição, onde a rotina, a alimentação e os meios de proteção contra infecções são os mesmos. Esse fato pode ser explicado pelos fatores intrínsecos ao desenvolvimento, especialmente destacado pela carga genética, pois, em igual ambiente, o desenvolvimento físico individual depende de fatores basicamente hereditários. Por outro lado, se tem demonstrado que crianças de distintas raças mostram curvas de crescimento semelhantes se as condições ambientais, a alimentação e a proteção contra as infecções são as mesmas $^{(9)}$.

Resultados diferentes foram encontrados no estudo transversal realizado com 1.386 crianças, menores de cinco anos, que residiam com suas famílias no estado de Alagoas, o qual apresenta os piores indicadores sociais do país; constatando a prevalência de déficits estaturais entre as crianças estudadas ${ }^{(11)}$.

Em contrapartida, o estudo transversal que avaliaram 33 crianças pré-escolares menores de 6 anos em uma creche do Rio de Janeiro constatou que nenhuma delas apresentou déficit pôndero-estatural e a sua maioria pertencia também à classe econômica desfavorável ${ }^{(12)}$.

As diferenças entre o presente estudo e o realizado no estado de Alagoas $^{(11)}$ permitem afirmar que as crianças abrigadas apresentaram índices de estatura superiores aos das crianças que participaram da pesquisa citada e que vivem com suas famílias, porém em condições sócio-econômicas desfavoráveis. Já no estudo em uma creche do Rio de Janeiro ${ }^{(12)}$ não houve alterações estaturais nas crianças pertencentes, em sua maioria, à classe desfavorável, o que comprova que o crescimento é influenciado por múltiplos fatores e que a influência ambiental sobre ele é variável. 
De acordo com a observação dos marcos do desenvolvimento propostos pelo Ministério da Saúde ${ }^{(1)}$, nas crianças abrigadas, foi verificado que em todas as faixas etárias, exceto entre 5 a 6 meses; pelo menos um marco não foi alcançado. Houve predomínio de déficits nos marcos sociais e psíquicos em todas as outras faixas etárias, pois entre as 44 crianças estudadas, 29 apresentaram pelo menos um desses marcos ausente. Já em relação aos marcos maturativos, 14 crianças demonstraram déficits, elas estavam na faixa etária de 2 a 6 anos. E quanto aos marcos psicomotores, apenas 3 crianças apresentaram dificuldades, e estavam entre 13 a 18 meses.

Resultados semelhantes foram encontrados no estudo transversal realizado em um abrigo da cidade de São Paulo com crianças de 2 a 6 anos de idade, através do qual foram analisadas habilidades funcionais de autocuidado, mobilidade e função social. Foi observado enquanto as habilidades motoras não tiveram déficits importantes, a função social foi significativamente mais prejudicada ${ }^{(13)}$.

Em contrapartida, um estudo longitudinal, realizado com crianças de 0 a 6 anos que frequentavam 3 creches da cidade de São Paulo, avaliou as habilidades na área de linguagem e pessoal-social, o qual foi observado um desenvolvimento positivo no aspecto social, já na área da linguagem não houve progressos significativos ao longo das avaliações ${ }^{(14)}$.

Percebe-se que a capacidade de interagir com o meio externo, de comunicar-se e de realizar tarefas que exigem raciocínio, que competem ao domínio social, estão prejudicadas nas crianças abrigadas, o que implica na necessidade de maior atenção nesses aspectos, que são fundamentais para o seu desenvolvimento. O contrário ocorreu na pesquisa realizada nas três creches da cidade de São Paulo que não apresentou déficits na socialização, pois as crianças viviam em família(14).

Sabe-se que as interações sociais e o ambiente familiar no qual a criança está inserida podem incentivar ou limitar tanto a aquisição de habilidades quanto a independência funcional. A qualidade das interações sociais nos abrigos necessita de transformações, visto que são notáveis os prejuízos no desenvolvimento dessas crianças, como o atraso na linguagem, que é influenciado pelas relações estabelecidas com as crianças ${ }^{(15)}$.

Pode-se constatar o quanto é importante para o desenvolvimento psíquico e social da criança a qualidade das relações estabelecidas com as pessoas que a cercam. Em um abrigo não há a presença materna ou paterna no cotidiano das crianças que lá vivem, e as pessoas que mais se aproximam dessa função são os cuidadores, que permanecem a maior parte do tempo com elas, participando efetivamente de todas as atividades, como alimentação, higiene, sono, lazer. Infelizmente, nos abrigos a relação entre a criança e o cuidador, torna-se, muitas vezes, despersonalizada, seja pela grande quantidade de crianças a serem cuidadas por cada um, ou pela própria falta de estímulos desse para com sua profissão.

Ainda em relação ao desenvolvimento psíquico, um estudo etnográfico realizado em um abrigo para crianças e adolescentes de 0 a 14 anos no interior de São Paulo comprova que a carência de relações afetivas e profundas entre as crianças institucionalizadas e seus cuidadores dificulta o estabelecimento de referências para seu desenvolvimento psíquico, sendo essa uma das causas para o seu desenvolvimento deficitário ${ }^{(16)}$.

Os resultados quanto à linguagem das crianças foi semeIhante ao estudo realizado em São Paulo ${ }^{(13)}$, em que se constatou ser comum encontrar crianças maiores de dois anos ainda emitindo apenas sons sem formar palavras.

Outro estudo que analisou o desenvolvimento de crianças entre 6 e 44 meses de idade em uma creche de São Paulo para famílias de baixa renda, demonstrou que a presença de fatores de risco psicossocial do ambiente em que as crianças viviam estava relacionada aos fatores de linguagem das crianças analisadas. À medida que os cuidadores, por problemas emocionais, não estão disponíveis para fornecer estímulos verbais para o desenvolvimento do vocabulário da criança, há um prejuízo no desenvolvimento da fala(17).

É de extrema importância que as dificuldades apresentadas pelas crianças quanto ao vocabulário, sejam trabalhadas pela equipe multiprofissional.

\section{CONCLUSÕES}

Foi percebido que a maioria das crianças apresentou o crescimento satisfatório comparando com os padrões adotados pelo Mistério da Saúde. No entanto, o desenvolvimento dessas crianças, em sua maioria apresentou atrasos importantes nos fatores sociais e psíquicos, dentre eles destaca-se a linguagem, já que houve déficit significativo desse domínio em todas as faixas etárias.

Somente realizando o acompanhamento do crescimento de maneira contínua será possível analisar as curvas dos gráficos de crescimento, se estão descendentes ou ascendentes, se estão indicando sinais de desnutrição ou de sobrepeso, permitindo também que haja uma ação preventiva eficaz.

No que se refere ao desenvolvimento infantil, é preciso que as crianças sejam assistidas continuadamente, de forma a trabalhar em seus sinais de atraso em quaisquer aspectos, já que a alteração em um deles pode interferir ao longo de suas vidas. Esse acompanhamento periódico das crianças permite que os profissionais da saúde realizem estratégias para favorecer o desenvolvimento neuro-psico-motor infantil.

Esse estudo possibilitou que houvesse uma reflexão sobre a maneira como as crianças residentes de abrigos estão sendo assistidas pela equipe de saúde, tornando-se necessário realizar o acompanhamento do crescimento e desenvolvimento dessas crianças, a fim de intervir sobre as alterações encontradas e de continuar esse processo de maneira regular.

Portanto, ressalta-se a importância da equipe de enfermagem durante o acompanhamento do crescimento e desenvolvimento infantil, no contexto da atenção primária à saúde, ao desempenhar ações para identificar precocemente alterações de saúde, direcionando as intervenções para a criação e implementação de estratégias de prevenção de doenças, manutenção e promoção da saúde, conforme as reais necessidades das crianças. 


\section{REFERÊNCIAS}

1. Ministério da Saúde. Secretaria de Políticas de Saúde. Saúde da criança. Acompanhamento do crescimento e desenvolvimento infantil. Brasília,DF: O Ministério; 2002.

2. Hockenberry MJ, Wilson D. Wong: fundamentos de enfermagem pediátrica. 8. ed. Rio de Janeiro: Elsevier; 2011.

3. Silva ERA, coordenadora. $O$ direito à convivência familiar e comunitária: os abrigos para crianças e adolescentes no Brasil. Brasília: IPEA/CONANDA; 2004.

4. Lima AKP, Lima AO. Perfil do desenvolvimento neuropsicomotor e aspectos familiares de crianças institucionalizadas na cidade do Recife. Rev CES Psicol 2012;5(1):11-25.

5. Freitas $\mathrm{HMB}$, Backes DS, Pereira ADA, Ferreira CLL, Marchiori MRC, Souza MHT, et al. Significados que os profissionais de enfermagem atribuem ao cuidado de crianças institucionalizadas com AIDS. Texto \& Contexto Enferm 2010;19(3):511-7.

6. Ministério da Saúde. Conselho Nacional de Saúde (Brasil). Resolução nº 196, de 10 de outubro 1996. Estabelece critérios sobre pesquisa envolvendo seres humanos. Diário Oficial da união 1996;Seção 1.

7. Ministério da Saúde (Brasil). Lei n. 8.069, de 13 de julho de 1990. Dispõe sobre o estatuto da criança e do adolescente e dá outras providências [legislação na internet]. Diário Oficial da União 16 jul 1990 [acesso em 25 jun 2012]. Disponível em: http://www.planalto.gov.br/ccivil 03/leis/l8069.htm

8. Abaid JLW, Dell'Aglio DD, Koller SH. Preditores de sintomas depressivos em crianças e adolescentes institucionalizados. Univ Psychol 2010;9(1):199-212.

9. Reichert APS, Almeida AB, Souza LC, Silva MEA, Collet N. Vigilância do crescimento infantil: conhecimento e práticas de enfermeiros da atenção primária à saúde. Rev RENE 2012;13(1):114-26.
10. Martino HSD, Ferreira AC, Pereira CNA, Silva R. Avaliação antropométrica e análise dietética de pré-escolares em centros educacionais municipais no sul de Minas Gerais. Ciênc Saúde Coletiva 2010;15(2): 551-58.

11. Ferreira HS, Luciano SCM. Prevalência de extremos antropométricos em crianças do estado de Alagoas. Rev Saúde Pública 2010;44(2):377-80.

12. Santos ALB, Leão LSCS. Perfil antropométrico de pré-escolares de uma creche em Duque de Caxias, Rio de Janeiro. Rev Paul Pediatr 2008;26(3):218-24.

13. Lanzillotta P, Rocha RP. Análise das habilidades funcionais em crianças em entidade filantrópica. Rev Soc Bras Clín Méd 2011;9(2):121-3.

14. Rezende MA, Beteli VC, Santos JLF. Avaliação de habilidades de linguagem e pessoal-sociais pelo Teste de Denver II em instituições de educação infantil. Acta Paul Enferm [periódico na internet]. 2005 Mar [acesso em 04 jul 2012];18(1):56-63. Disponível em: http:// www.scielo.br/scielo.php?script $=$ sci_arttext\&pid $=$ S0103-21002005000100008

15. Moré CLOO, Sperancetta A. Práticas de pais sociais em instituições de acolhimento de crianças e adolescentes. Psicol Soc 2010;22(3):519-28.

16. Barros RC, Fiamenghi Júnior GA. Interações afetivas de crianças abrigadas: um estudo etnográfico. Ciênc Saúde Coletiva 2007;(12)5:1267-76.

17. Maria-Mengel MRS, Linhares MBM. Fatores de risco para problemas de desenvolvimento infantil. Rev Latino-Am Enferm [periódico na nternet]. 2007 Out [acesso em 04 jul 2012];15(nº. esp.):837-42. Disponível em: http://www.scielo.br/scielo.php?pid =S0104$-11692007000700019 \&$ script $=$ sci_arttext\&tlng $=$ pt 